\title{
Control of Thermal Deflection in Concrete Structures Using Iron- Based Shape Memory Alloys
}

\author{
Brady Edmiston, Allen Davis, Mirmilad Mirsayar, and Darren Hartl* \\ Department of Aerospace Engineering, Texas A\&M University, College Station, Texas, USA
}

\begin{abstract}
Mitigating the structural damage brought about by thermal expansion is a primary objective in the design of vital concrete infrastructures, such as bridges or buildings. Shape memory alloys (SMAs), through their ability to recover strains through thermal loading-induced phase transformations, offer a distinct advantage in achieving this design goal as such strain recovery in embedded components could be used to oppose thermal expansion in a surrounding matrix (e.g. concrete). This study seeks to characterize the thermal expansion of system, comprised of an SMA rod embedded in a concrete block undergoing a thermal loading cycle. Characterization is produced through a full factorial analysis, wherein evaluation is performed through the Abaqus unified finite element analysis suite. This preliminary analysis indicates that, while iron-based SMAs show promise in this field due to their low manufacturing costs, their large thermal hysteresis may lead to limited phase transformation in this application.
\end{abstract}

\section{Introduction}

All concrete structures are subjected to thermal loading cycles throughout their lifetime. These cycles can be from small, daily temperature changes or from larger, seasonal weather patterns. Temperature change on any scale causes thermal deflections, which can cause problems in structures such as cracking and misalignment, and may ultimately lead to structural failure in infrastructure used by thousands of people each day. When temperature gradients are taken into account, the difference in temperature between the top and bottom surfaces of concrete slabs can cause curling. This curling results in stresses within the slab, which will lead to structural degradation [1]. However, this paper focuses on translational deflections caused by uniform thermal cycling and leaves the issue of temperature gradients for future work.

To prevent structural deterioration, innovative and adaptive solutions are needed. Fortunately, a likely candidate, shape memory alloys (SMAs), have been at the forefront of research efforts in recent years. SMAs are a polymorphic, "smart" or "multifunctional" material that possess a key and unique phenomenon that give them a distinct advantage over the traditional steel structural supports used in concrete infrastructure today: the shape memory effect [2]. This effect is the result of a material phase transformation from the high-stress, lowtemperature martensitic phase to the low-stress, hightemperature austenite phase, in which the specimen can fully recover the seemingly permanent strains [3]. This ability to recover strains through a change in temperature, as demonstrated in Figure 1, is what makes SMAs a desirable solution to this application since such a recovery, in the context of uniaxial rods, can be used to oppose the expansive tendencies of a surrounding matrix during heating.

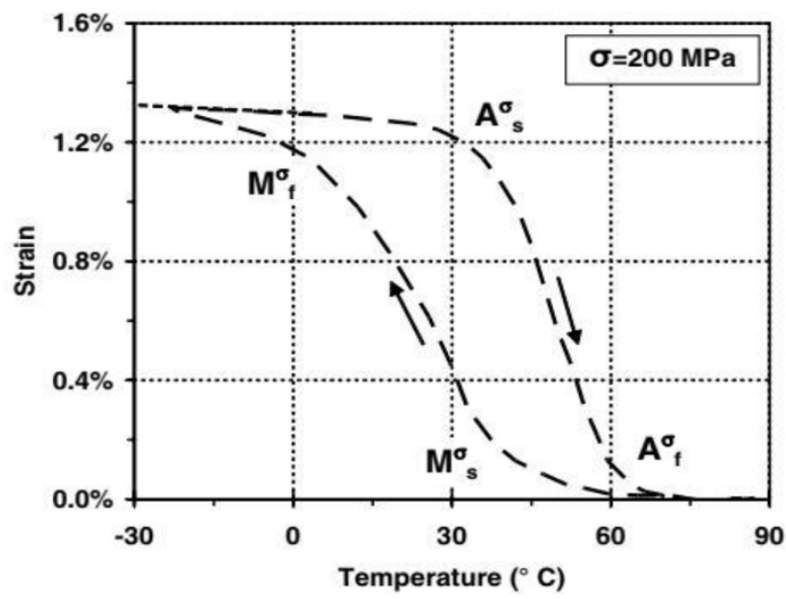

Fig. 1. Temperature-Strain cycle of an SMA sample (Reprinted: [5]).

Most research on SMA applications to date has been conducted over the Ni-Ti alloy, commonly referred to as Nitinol or NiTi. However, the high material and processing costs severely limit any commercial application of this alloy, as any system designed to mitigate thermal expansion in civil infrastructure must be scalable so that it is applicable to a plethora of configurations and environments. As an alternative, ironbased SMAs (Fe-SMAs) appear very promising thanks to their low cost and high elastic stiffness [4]. Therefore, a study of the effects of Fe-SMAs and their material properties on a concrete system through numerical

\footnotetext{
* Corresponding author: darren.hartl@tamu.edu
} 
analysis is needed to determine the applicability of FeSMAs as a deterrent to structural damage brought about by thermal expansion.

\section{Evaluation}

\subsection{Boundary value problem}

To model the problem, the finite element method is employed through finite element software, Abaqus. The Abaqus model is comprised of a concrete block with an embedded Fe-SMA rod, as shown with the given dimensions in Figure 2. Table 1 defines the constant material properties along with specimen geometries used in the analysis. The Fe-SMA is considered to have a reference (initial) state such that it is in the martensite phase and gas prestrained to $H_{\max }^{t}$ (e.g., the maximum strain it can recover during heating). The concrete is encastered on one end while free on the other. The interaction between the concrete and the Fe-SMA rod is approximated using Abaqus's "embed" constraint, with the concrete being the "host" and the Fe-SMA rod being the "embedded elements" [6] The embed constraint constrains the translational degrees of freedom of the embedded elements' nodes to that of the host region. One limitation of this approximation is that it cannot consider "slip" between the concrete and Fe-SMA rod. To address this, two different failure criteria were assessed. In the first, the end of the Fe-SMA rod is "anchored" to the exposed surface of the concrete such that debonding between the Fe-SMA rod and concrete is not a concern, making the only failure mode assessed yielding of the rod.
In the second, the Fe-SMA rod is connected to the concrete only along its length (e.g., in a cohesive manner, modeled using the "embed" option), and failure is defined as either yielding of the rod or stress in the concrete exceeding $\sigma_{c}^{\text {ult }}$. The thermal cycle modeled is outlined in Table 2. The thermal cycle heating and cooling values were determined based on bridge design specifications for Dallas, TX [7], and as such the heating and cooling cycle is driven by the local environment. An output of particular interest in the cycle analysis is the total composite block axial strain after heating, $\epsilon_{\text {heat }}^{c}$.

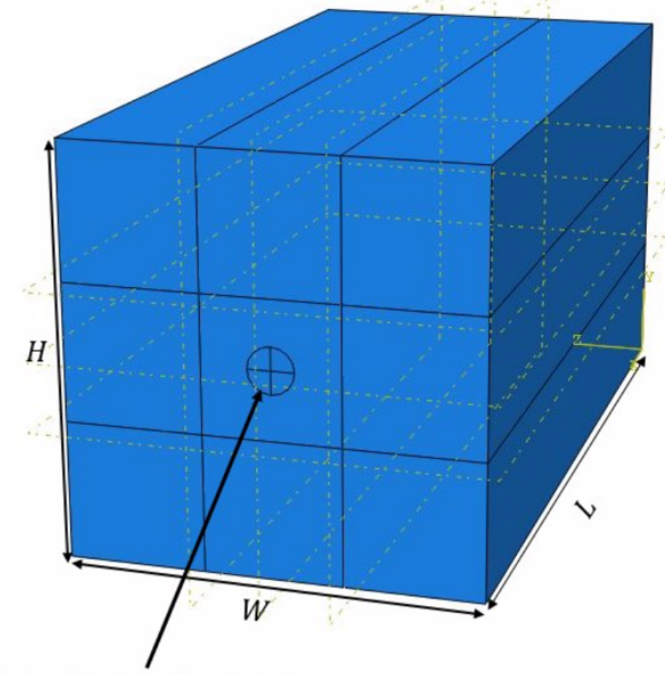

Embedded Fe-SMA Rod

Fig. 2. The system as modeled in Abaqus.

Table 1. Constant Analysis Parameters, [7-9].

\begin{tabular}{|c|c|c|c|c|c|}
\hline Parameter & Description & Value & Parameter & Description & Value \\
\hline$W$ & Concrete width & $15 \mathrm{~mm}$ & $H$ & Concrete height & $15 \mathrm{~mm}$ \\
\hline$L$ & Concrete/Rod length & $30 \mathrm{~mm}$ & $E_{\text {concrete }}$ & Concrete elastic modulus & $4.8 \mathrm{E}+3 \mathrm{MPa}$ \\
\hline$v_{\text {concrete }}$ & Concrete Poisson's ratio & 0.2 & $\alpha_{\text {concrete }}$ & $\begin{array}{c}\text { Coefficient of thermal } \\
\text { expansion of concrete }\end{array}$ & $1.4 \mathrm{E}-41 / \mathrm{K}$ \\
\hline$M_{s}$ & Martensite start temperature & $310 \mathrm{~K}$ & $M_{f}$ & Martensite finish temp & $270 \mathrm{~K}$ \\
\hline$A_{f}$ & Austenite finish temperature & $335 \mathrm{~K}$ & $A_{s}$ & Austenite start temperature & $300 \mathrm{~K}$ \\
\hline$E_{M}$ & Martensite elastic modulus & $50 \mathrm{E}+3 \mathrm{MPa}$ & $E_{A}$ & Austenite elastic modulus & $100 \mathrm{E}+3 \mathrm{MPa}$ \\
\hline$T_{h}$ & Heating temperature & $321 \mathrm{~K}$ & $T_{s}$ & Start temperature & $296 \mathrm{~K}$ \\
\hline$v_{S M A}$ & SMA Poisson's ratio & 0.33 & $T_{c}$ & Cooling temperature & $271 \mathrm{~K}$ \\
\hline$\sigma_{c}^{\text {ult }}$ & Ultimate stress of concrete & $3.24 \mathrm{MPa}$ & - & - & - \\
\hline
\end{tabular}

Table 2. Thermal Cycle in Abaqus.

\begin{tabular}{|c|c|}
\hline Step Number & Process \\
\hline Step-0 & Initialize analysis, $T=T_{s}$. \\
\hline Step-1 & Heat to $T_{h}$. \\
\hline Step-2 & Cool to $T_{s}$. \\
\hline Step-3 & Cool to $T_{c}$. \\
\hline Step-4 & Heat to $T_{s}$. \\
\hline
\end{tabular}

\subsection{Design of experiments (DOE)}

To quantify the Fe-SMA effect on the system, a full factorial analysis is performed, whereby all possible combinations of design variables with one another at each level are considered. For this study, five design variables (detailed in Table 3) were evaluated at three levels each, equating to a total of 256 functional evaluations (i.e., FEA runs). Once the data is produced, it is assessed through factor effects plots using the analysis of means. These 
plots allow each design variable's effect on each output variable to be easily visualized.

\section{Results}

\subsection{DOE results and discussion}

Figure 3 is a plot of the temperature-strain curve of an sample design against the Control Case, which is defined as simply a concrete block, having a coefficient of thermal expansion of $\alpha_{\text {concrete, }}$ with no structural support. $\sigma_{\text {heat }}^{c}$ is measured from the zero-strain axis. Here we see that, in the case that the Fe-SMA is considered, the iniation of transformation during heating serves to reduce the axial strain expansion. This effect is also exhibited, albeit to a lesser degree, during cooling. The factor effects plot is given in Figure 4. This graph shows the average value of the output variable at each non-dimensional level of the design variables. For this data, what is important is the slope of the lines, not necessarily the values themselves since it is based on an average value. The steeper the slope, the greater the relative impact of that design variable on the output variable. Figure 5 shows the temperature-stress curve of a sample design over a phase diagram of the material used in that design.

Table 3. Design variables and their bounds [2, 10-13].

\begin{tabular}{|c|c|c|}
\hline $\begin{array}{c}\text { Design } \\
\text { Variable }\end{array}$ & Description & Bounds \\
\hline$H_{\text {max }}^{t}$ & $\begin{array}{c}\text { Max transformation } \\
\text { strain of the Fe-SMA } \\
\text { rod. }\end{array}$ & $0.034-0.067$ \\
\hline$\alpha$ & $\begin{array}{c}\text { Thermal expansion } \\
\text { coefficient of Fe-SMA } \\
\text { rod, both phases. }\end{array}$ & $16.5 E^{-6}-22.0 E^{-6} \frac{1}{K}$ \\
\hline$C_{m}$ & $\begin{array}{c}\text { Martensitic stress } \\
\text { influence coefficient. }\end{array}$ & $0.53-3.1 \mathrm{MPa} / \mathrm{K}$ \\
\hline$C_{a}$ & $\begin{array}{c}\text { Austenite stress } \\
\text { influence coefficient. }\end{array}$ & $0.53-3.1 \mathrm{MPa} / \mathrm{K}$ \\
\hline$R o d V F$ & $\begin{array}{c}\text { Volume fraction of } \\
\text { rod to concrete. }\end{array}$ & $0.01-0.05$ \\
\hline
\end{tabular}

It can be seen from Figure 4 that the Austenite stress influence coefficient, $C_{a}$, certainly has the largest overall effect on the system [14]. Since the Fe-SMA is initially fully martensitic, it will phase shift into austenite as the temperature approaches $A_{s}$. The low range of $C_{a}$ values used means that the stress-temperature transformations are more sensitive to stresses in the rod. At the same time, $C_{m}$ has a low impact on the output variable. This is due to the fact that $\epsilon_{\text {heat }}^{c}$ is entirely dependent upon the strain recovery from the austenite phase transformation.

As shown in Figure 3, during the heating phase, the presence of the rod lowers the strain, which is the key focus of this study. However, after completing the cycle there is a residual strain present at the tip of the concrete block. In infrastructure, this could lead to large gaps in the structure, creating openings for water or other forms of damage. To prevent this, larger transformations must be achieved during the cooling phase of this system. In this study, the phase transformation temperatures $\left(A_{s}, A_{f}, M_{s}\right.$,
$M_{f}$ ) were treated as constant, and were spaced out in agreement with the large thermal hysteresis of Fe-SMAs, which limited the overall phase transformations achieved. Alternatively, one could incorporate this unrecovered strain into the design of a structure by cycling rod elements once before implementation into the matrix bed.

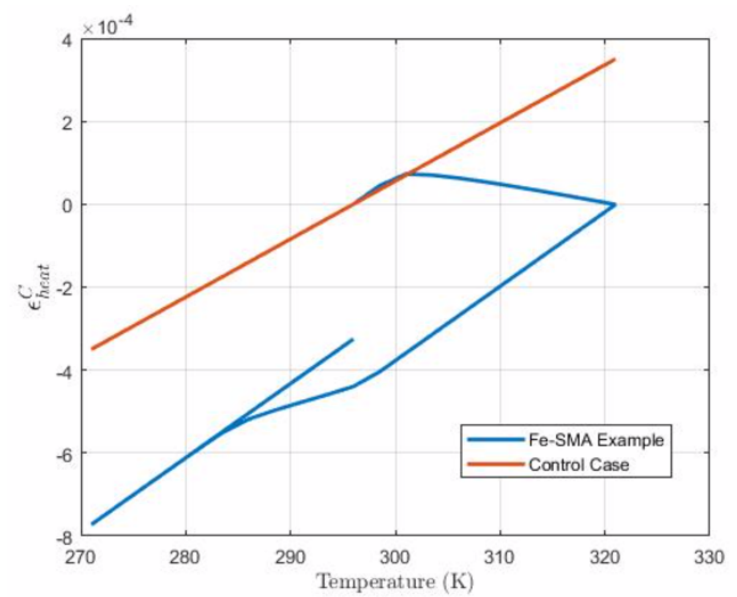

Fig. 3. Sample design's temperature-strain curve plotted against the control case. The Fe-SMA reduces axial strain during expansion, shifting the curve downwards due to the Fe-SMA.

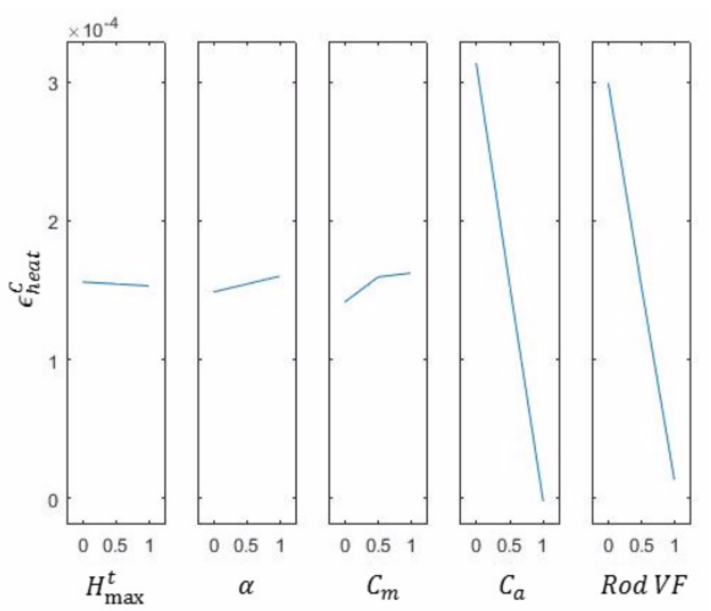

Fig. 4. Factor effects plots showing the influence of each design variable on the output $\epsilon_{\text {heat }}^{c}$ (the strain at the tip of the concrete at the end of the heating phase). Note that the slopes are demonstrative of the design variable's relative effect on the output variable, and the actual values are not as relevant.

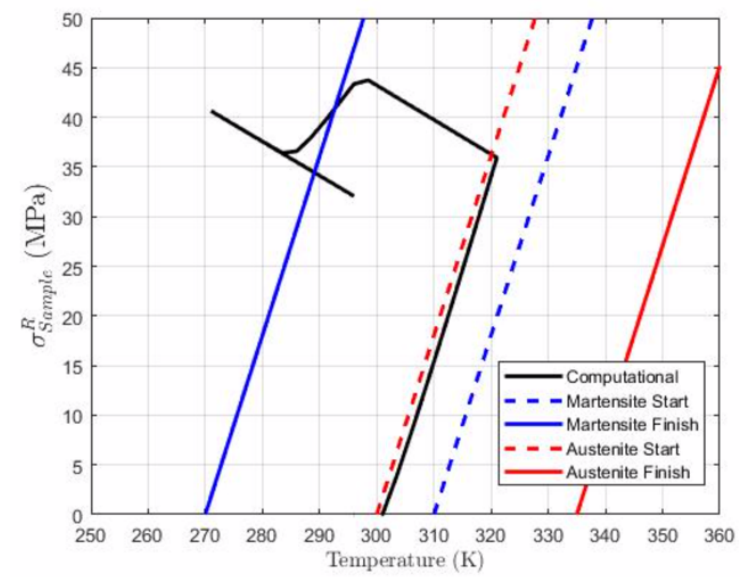


Fig. 5. Sample design's stress-temperature curve plotted along with the phase diagram of the Fe-SMA modeled. T slopes of the red lines are $C_{a}$ and the slopes of the blue lines are $C_{m}$.

All results shown are for the failure criteria of the anchored rod case. When limiting the stress in the rod to $\sigma_{c}^{u l t}$, it was found that this constraint is exceeded easily, and the designs would fail before making any noticeable difference in the strain of the rod. Thus, specific results for those cases need not be reported.

\section{Conclusions}

Fe-SMAs show promise as a method to mitigate structural damage in infrastructure. Their shape memory effect allows them to recover strains well enough that an FeSMA rod is able to counter the thermal expansion of a concrete matrix that it was embedded within. This performance in a heating cycle is encouraging for FeSMA's future applicability to this problem, however it was demonstrated that in the complete heating/cooling cycle the rod lead to a larger deformation in the cooling phase, opening up more possibilities for damage. Two solutions are proposed. First, reducing the thermal hysteresis of Fe-SMAs as a critical step in implementing them as structural supports. This reduction will allow the material to complete more of the phase transformations, thereby lowering the strains in both the heating and cooling phases. Second, this unrecovered strain could be accounted for in the design of the structure by cycling rod elements (thereby introducing the strain) before installation into the matrix. Finally, studies of the effects of both cohesive interaction between the concrete and rod in the proposed model and the aptitude of Fe-SMAs in controlling curling in concrete slabs due to thermal gradients are needed to further assess iron-based SMAs in this application.

\section{References}

1. Mirsayar, M.M., Huang, K., and Zollinger, D.G. (2016). New Approach to Determining Concrete Slab Lift-Off by Use of Interfacial Fracture Mechanics Concepts. Transportation Research Record: Journal of the Transportation Research Board, 2590, 10-17.

2. Janke, L., Czarderski, C., Motavalli, M., and Ruth, J. (2005). Application of Shape Memory Alloys in Civil Engineering Structures-Overview, Limits, and New Ideas. Materials and Structures, 38, 578-502.

3. Hartl, D.J., and Lagoudas, D.C. (2007). Aerospace applications of shape memory alloys. Proceedings of the Institution of Mechanical Engineers, Part G: Journal of Aerospace Engineering, 221, 535-552.

4. Czaderski, C., Shahverdi, M., Brönnimann, R., Leinenbach, C., and Motavalli, M. (2014). Feasibility of iron-based shape memory alloy strips for prestressed strengthening of concrete structures. Construction and Building Materials, 56, 94-105.

5. Lagoudas, Dimitris C. (2011). Shape Memory Alloys: Modeling and Engineering Applications. Springer.
6. Smith M. (2009). ABAQUS/Standard User's Manual, Version 6.9. Providence, RI: Simula.

7. AASHTO LRFD bridge design specifications, Part I: Sections 1-6, Washington, DC: American Association of State Highway and Transportation Officials, 2017.

8. Lecce, L. (2015). Shape memory alloy engineering: for aerospace, structural and biomedical applications, Amsterdam: Elsevier.

9. Omori, T., Ando, K., Okano, M., Xu, X., Tanaka, Y., Ohnuma, I., Kainuma, R., and Ishida, K. (2011). Superelastic Effect in Polycrystalline Ferrous Alloys. Science, 333, 68-71.

10. Cladera, A., Weber, B., Leinenbach, C., Czaderski, C., Shahverdi, M., and Motavalli, M. (2014). Ironbased shape memory alloys for civil engineering structures: An overview. Construction and Building Materials, 63, 281-293.

11. Lee, H.J., and Lee, J.J. (2000). A numerical analysis of the buckling and postbuckling behavior of laminated composite shells with embedded shape memory alloy wire actuators. Smart Materials and Structures, 9, 780-787.

12. Qidwai, M.A.S., Hartl, D.J., and Lagoudas, D.C. (2000). Numerical Implementation of an SMA Thermomechanical Constitutive Model Using Return Mapping Algorithms. Shape Memory Alloys, 47, 189-231.

13. Soufeiani, L., Raman, S.N., Jumaat, M.Z.B., Alengaram, U.J., Ghadyani, G., and Mendis, P. (2016). Influences of the volume fraction and shape of steel fibers on fiber-reinforced concrete subjected to dynamic loading - A review. Engineering Structures, 124, 405-417.

14. Lagoudas, D., Hartl, D., Chemisky, Y., Machado, L., and Popov, P. (2012). Constitutive model for the numerical analysis of phase transformation in polycrystalline shape memory alloys. International Journal of Plasticity, 32, 155-183. 